\title{
Chaotic Correlation Functions with Complex Fermions
}

\author{
Ritabrata Bhattacharya $^{a}$, Dileep P. Jatkar ${ }^{a}$, Arnab Kundu ${ }^{b}$ \\ ${ }^{a}$ Harish-Chandra Research Institute, Homi Bhabha National Institute (HBNI) \\ Chhatnag Road, Jhunsi, Allahabad 211 019, India \\ ${ }^{b}$ Theory Division, Saha Institute of Nuclear Physics, Homi Bhabha National Institute (HBNI) \\ 1/AF Bidhannagar, Kolkata 700064, India \\ E-mail: ritabratabhattacharya[at]hri.res.in, dileep[at]hri.res.in, \\ arnab.kundu [at] saha.ac. in
}

Abstract: We study correlation functions in the complex fermion SYK model. We focus, specifically, on the $h=2$ mode which explicitly breaks conformal invariance and exhibits the chaotic behaviour. We explicitly compute fermion six-point function and extract the corresponding six-point OTOC which exhibits an exponential growth. Following the program of Gross-Rosenhaus, we estimate the triple short time limit of the six point function. Unlike the conformal modes with high values of $h$, the $h=2$ mode has contact interaction dominating over the planar in the large $q$ limit. 


\section{Contents}

1 Introduction $\quad 1$

2 SYK model with complex fermions $\quad 5$

2.1 Large $q$ limit 6

3 Correlation Functions $\quad 7$

4 Away from the Conformal Limit $\quad 10$

4.1 The enhanced four point contribution $\quad 12$

4.2 The "Contact" and and "Planar" diagrams 12

4.3 The six point function 14

4.4 The Short time and OTO behavior of the Six point function 15

$\begin{array}{lll}5 & \text { Conclusion } & 17\end{array}$

$\begin{array}{lr}\text { A Appendix A } & 18\end{array}$

$\begin{array}{ll}\text { B Appendix B } & 18\end{array}$

\section{Introduction}

A generic dynamical system is inherently chaotic[1]. For classical systems, chaos can be easily characterized by the sensitivity of trajectories with respect to initial conditions. For quantum systems, lacking in the concept of trajectories, the notion of chaos is more subtle. Often, quantum chaos can be characterized in terms of properties of the spectrum of the Hamiltonian. In the semi-classical approach, there is a relatively simple definition of chaotic behaviour, directly adopted from the sensitivity of classical trajectories with respect to initial conditions.

For classical dynamical systems, characterized by phase space coordinates $\{q(t), p(t)\}$, where $q(t)$ and $p(t)$ are generalized positions and generalized momenta. A particular trajectory is 
represented by $q(t)$. High sensitivity of the late time trajectory with respect to the initial condition can be quantified as:

$$
\exp \left(\lambda_{\mathrm{L}} t\right)=\frac{\partial q(t)}{\partial q(0)} \equiv\{q(t), p(t)\}
$$

where $\lambda_{\mathrm{L}}$ is the so-called Lyapunov exponent and the right-most expression above is the Poisson bracket[1]. By virtue of the correspondence principle, we obtain a quantum mechanical characterization, by replacing the Poisson bracket with a commutator: $\{q(t), p(t)\} \rightarrow$ $-i \hbar[q(t), p(t)][2]$. Instead of computing the commutator, one calculates the squared commutator, so that there is no spurious cancellation due to destructive phases. This argument, however, is limited and does not necessarily imply that allowing for such phases will always cancel the chaotic growth. In this article, we will calculate the cubic power of the commutator, which will explicitly display the exponential growth behaviour.

Thus, we can define a generic function for the diagnostic of chaos:

$$
C_{(n)}\left(t_{1}, t_{2}\right) \equiv\left\langle\left[V\left(t_{1}\right), W\left(t_{2}\right)\right]^{n}\right\rangle
$$

where $n \in \mathbb{Z}_{+}$, and $V$ and $W$ are two self-adjoint operators and the expectation value is defined with respect to a particular state of the system. Note that, in defining the chaos diagnostic in (1.2), we have recast the chaotic property as a feature of $n$-point correlation function of the system. A straightforward analogy with the classical limit does not preclude a two-point function from displaying the exponential growth, but we know of no explicit example of the same. In this article, we will explicitly discuss the case for $n=3$ in a thermal state.

Before doing so, let us briefly look at the $n=2$ case. Written explicitly, the commutator contains various four-point functions with no particular time-ordering, since $t_{1}$ and $t_{2}$ are defined without any ordering. For a thermal state expectation value, using the KMS conditions ${ }^{1}$, it is further possible to rearrange the various four-point functions in terms of two pieces: one timeordered four-point function and another out-of-time-ordered correlator (OTOC). These are given by $\langle V(0) V(0) W(t) W(t)\rangle$ and $\langle V(0) W(t) V(0) W(t)\rangle$, respectively, choosing $t_{1}=0$ and $t_{2}=t$. The time-ordered correlator does not display the exponential growth, it is contained in the four-point OTOC.

For $n=3$, upon using the KMS condition, the chaos diagnostic in (1.2) has one time-ordered and two OTOC pieces. These are simply, $\langle V(0) V(0) V(0) W(t) W(t) W(t)\rangle$ (time-ordered) and $\langle V(0) W(t) V(0) W(t) V(0) W(t)\rangle,\langle V(0) W(t) V(0) V(0) W(t) W(t)\rangle,\langle W(t) V(0) V(0) W(t) V(0) W(t)\rangle$, etc, which are OTOC. While a complete understanding of the behaviour of (1.2) for arbitrary

\footnotetext{
${ }^{1} \mathrm{KMS}$ condition is simply the Euclidean periodicity condition on thermal correlators. For example, for two operators $V(0)$ and $W(t)$, the KMS condition on the two-point function reads:

$$
\operatorname{tr}\left(e^{-\beta H} W(t) V(0)\right)=\operatorname{tr}\left(e^{-\beta H} V(0) W(t+i \beta)\right) .
$$

Here $\beta$ is the inverse temperature. Evidently, this condition can be used to interchange the order of the operators inside a thermal correlator.
} 
$n$ is desirable, we will explore an exact calculation for $n=3$ in this article, with a particularly simple model.

The model we consider is a simple generalization of the so-called Sachdev-Ye-Kitaev (SYK) system[3-6], in which one considers fermionic degrees of freedom with an all-to-all interaction. The interaction coupling is drawn from a random Gaussian distribution with a zero mean value and a given width. In the large $N$ limit, in which the number of fermionic degrees of freedom becomes infinite, the system becomes analytically tractable in the sense that the corresponding Schwinger-Dyson equations can be explicitly determined. The solution of this equation readily determines the two-point function, as a function of the coupling strength, in general. In particular, in the low energy limit, this Schwinger-Dyson equation is analytically solvable and yields a two-point function with a manifest $\mathrm{SL}(2, R)$ symmetry. In the infra-red (IR), this is described by a conformal field theory (CFT), and the two-point function breaks the conformal group into the $\mathrm{SL}(2, R)$ subgroup. In the large $N$ limit, further, the four-point correlator can be explicitly calculated, which yields the corresponding Lyapunov exponent: $\lambda_{\mathrm{L}}=2 \pi T$, where $T$ is the temperature of the thermal state. Here, we are working in natural units. This Lyapunov exponent saturates the so-called chaos bound[7]. Intriguingly, the chaos bound saturation also occurs for black holes, in which the local boost factor at the event horizon determines the corresponding Lyapunov exponent as well as the corresponding Hawking temperature. Only extremal black holes have an $\operatorname{SL}(2, R)$ global symmetry, due to the existence of an $\mathrm{AdS}_{2}$ sector near the horizon. Correspondingly, the low energy conformal system coming from the SYK model can be shown to capture the essential physics of the $\mathrm{AdS}_{2}[8]$.

The low energy effective action for the SYK model is simply given by a Schwarzian effective action, which can also be shown to arise from the two-dimensional Jackiw-Teitelboim theory in $[8,9]$ (In fact, exact derivation of the Schwarzian effective action has been done using the fluid/gravity correspondence[6]). However, in this context, the non-trivial statements of holography necessitates keeping a leading order correction away from the purely $\mathrm{AdS}_{2}$ throat, as well as from the purely $\mathrm{CFT}_{1}$ in the IR, hence it goes by the acronym of NAdS/NCFT. From the geometric perspective, $\mathrm{AdS}_{2}$ appears in the following two cases: (i) in the extremal limit of a black hole in asymptotically flat background, (ii) in the deep IR of an asymptotically $\mathrm{AdS}_{d+1^{-}}$ background. Often, in the second case, the deep IR results from an RG-flow connecting a $\mathrm{UV} \mathrm{CFT}_{d}$ to an IR $\mathrm{CFT}_{1}$, as a result of a relevant density perturbation in the UV CFT. Holographically, such operators correspond to turning on a bulk U(1)-flux, in the simplest case. A standard example of this is the AdS-Reissner-Nordstrom black hole: It asymptotes to an AdS geometry and the extremal limit consists of an $\mathrm{AdS}_{2}$ in the IR, which is supported by the flux. In the $\mathrm{AdS}_{2}$ throat, the flux is a simple scalar field, and the boundary theory has a natural notion of a conserved charge and therefore a non-vanishing chemical potential.

The SYK model which is defined with $N$ Majorana fermions, $\psi^{i}(i=1, \ldots, N)$ in $(0+1)$ dimensions, cannot have a charge density and correspondingly a non-vanishing chemical potential. If we, instead, consider $N$ complex fermions, $\psi_{i}$ and $\psi_{i}^{\dagger}$, it is natural to introduce a $\psi_{i}^{\dagger} \psi_{i}$ term in the Lagrangian, with chemical potential as the coupling. Similar to the SYK 
model, we consider a $q$-body interaction term, with $q / 2$ number of $\psi$ and $q / 2$ number of $\psi^{\dagger}$, whose couplings are chosen from a Gaussian distribution, with a standard deviation denoted by $J$. In the limit $q \gg 1$, this system becomes exactly solvable in an (1/q)-expansion[4], which is what we will use in evaluating the explicit correlation functions. Motivated from the previous paragraph, it is therefore natural to consider a particular generalization of the SYK model with a $U(1)$ global symmetry.

The standard SYK theory, with Majorana fermions, has a particular operator at the conformal fixed point, whose four point OTOC displays chaotic behavior with Lyapunov exponent $\lambda_{L}=$ $\frac{2 \pi}{\beta}[3-6,10-37]$. The generalization of this model to complex fermions was done in [38-40]. In [38], the low energy effective action of an SYK-model with complex fermions was discussed. It was shown that the presence of a non-vanishing chemical potential does not break the conformal symmetry in the deep IR, as long as one amends the conformal transformations with a gauge transformation. Thus, the resulting low energy effective action is simply a Schwarzian action along with a free bosonic theory with a standard kinetic term[14, 17]. Therefore, from a strict IR perspective, the maximal chaos holds for any non-vanishing value of the chemical potential.

The non-triviality comes from the order of limits. The exponential growth of the four-point OTOC holds for a larger regime compared to the long-time (and therefore, deep IR) limit. For sufficiently large time, one recovers the maximal chaos. However, there exists an intermediate regime in which the exponential growth takes place with a different Lyapunov exponent. This is physically equivalent to staying in a medium energy scale and finding a chaotic behaviour of the correlation function at this energy scale. This associates naturally an RG-flow of the Lyapunov exponent itself.

In the standard SYK model, in the large $q$ limit, the relevant scale in the system is provided by an effective coupling:

$$
\mathcal{J}=\frac{q J^{2}}{2^{q-1}},
$$

which has mass dimension one. The IR CFT resides in the $\mathcal{J} \rightarrow \infty$ limit, but the exponential growth of OTOC and subsequently the Lyapunov exponent can be obtained as a perturbation series in $1 / \mathcal{J}$. This naturally gives an RG-flow of the Lyapunov exponent[4]. In [40] we studied the SYK model with complex fermions in the large $q$ limit in the presence of a chemical potential $\mu$. Here, in the UV Hamiltonian, we have two natural parameters: $\beta \mu$ and $\beta \mathcal{J}$ and the effective coupling in the IR is given by

$$
\mathcal{J}_{\text {eff }}^{2}=\frac{q}{2} \frac{J^{2}}{(2+2 \cosh (\mu \beta))^{\frac{q}{2}-1}} .
$$

The strict IR is located at $\mathcal{J}_{\text {eff }} \rightarrow \infty$ limit, and one can calculate systematically the RG-flow of the Lyapunov exponent in a perturbation series in $1 / \mathcal{J}_{\text {eff }}$. This RG-flow shows sensitive behaviour for the Lyapunov exponent as the UV parameter $\beta \mu$ is dialled up[40].

In keeping with the theme, in this article, we further compute higher point OTOC for complex fermion SYK-model, with a non-vanishing chemical potential. Our analyses follow closely the 
analyses in [41], in the large $q$ limit. However, our analyses are performed in the complementary regime in that we completely focus on the operators that display chaotic nature and away from the conformal limit. In spirit of the NAdS/NCFT picture, this is rather natural regime to consider; in the context of chaotic properties of many body systems, this is an example of a tractable and explicit higher point OTOC which displays the expected exponential growth.

In this paper, after computing the fermion six point function with a non-vanishing chemical potential, we take the triple short time limit to estimate the the bulk three point correlator, away from the conformal limit. In this regard, we compute bulk three point function(triple short time limit of the fermion six point correlators, neglecting the Schwarzian mode) of the modes satisfying conformal invariance as well as the Schwarzian mode, using the techniques employed by Gross and Rosenhaus[41].

This paper is organized as follows. In Section 2, we briefly review the SYK model with complex fermions. In Section 3, we compute the six point fermion correlator in the triple short time limit. We then interpret it in terms of the bulk three point correlator in the IR limit of the conformal modes and check that we do indeed find them to be of the form of conformal three-point function, in the triple short time limit. We apply this technique in Section 4 to compute the six point function and take the triple short time limit to determine the three point correlation function of fermion bilinears away from the conformal limit. We carry out this computation in the presence of a chemical potential $\mu$. We conclude with the discussion of our results, and possible future directions.

\section{SYK model with complex fermions}

The SYK model with complex fermion in $0+1$ dimensions is defined by the Hamiltonian with all to all random interaction between $q$ fermions,

$$
H=\sum J_{i_{1} i_{2} \cdots i_{q / 2} i_{q / 2+1} \cdots i_{q}} \psi_{i_{1}}^{\dagger} \psi_{i_{2}}^{\dagger} \cdots \psi_{i_{q / 2}}^{\dagger} \psi_{i_{q / 2+1}} \cdots \psi_{i_{q}} .
$$

An exhaustive study of this model is done in [39], we will mention some of the essential features that will be necessary for our analysis. In addition to the higher dimensional operators of the form $\mathcal{O}_{n}=\frac{1}{N} \sum_{i} \psi_{i}^{\dagger} \partial_{t}^{2 n+1} \psi_{i}$ which behave in a manner similar to those found in the SYK model with Majorana fermions; we also have the operators of the form $\tilde{\mathcal{O}}_{n}=\frac{1}{N} \sum_{i} \psi_{i}^{\dagger} \partial_{t}^{2 n} \psi_{i}$. The lowest lying mode of these operators give the Schwarzian mode and the $U(1)$ charge respectively. In absence of a mass like term in the action the two point function of the particle and anti-particle are the same in the free case as well as the low energy limit of the interacting theory.

$$
G_{\text {free }}(\tau)=\frac{1}{2} \operatorname{sgn}(\tau), \quad G_{c}(\tau)=b \frac{\operatorname{sgn}(\tau)}{|\tau|^{\frac{2}{q}}}
$$

where, $G_{c}(\tau)$ is the propagator in the conformal limit. In the low energy, i.e., IR limit it is possible to obtain the four point function of the fermions using the expansion in the eigenbasis of the quadratic Casimir operator. Skipping the details we state here only the results. 
Since we have complex fermions, i.e., $\psi_{i}=\xi_{i}+\mathbf{i} \eta_{i}$ in case of the correlation functions we have contribution of two different kinds,

$$
\left\langle\psi^{\dagger}\left(t_{1}\right) \psi\left(t_{2}\right) \ldots\right\rangle=\left\langle\left(\xi\left(t_{1}\right) \xi\left(t_{2}\right)+\eta\left(t_{1}\right) \eta\left(t_{2}\right)\right) \ldots\right\rangle+\mathbf{i}\left\langle\left(\xi\left(t_{1}\right) \eta\left(t_{2}\right)-\eta\left(t_{1}\right) \xi\left(t_{2}\right)\right) \ldots\right\rangle .
$$

While the first piece, namely, the real part is anti-symmetric under the exchange of $t_{1}$ and $t_{2}$, the second piece is symmetric.

In case of the four point function if we consider the time reversal invariant contribution this leads to two different contributions namely $F^{A}\left(\tau_{1}, \tau_{2}, \tau_{3}, \tau_{4}\right)$ and $F^{S}\left(\tau_{1}, \tau_{2}, \tau_{3}, \tau_{4}\right)$ which are respectively anti-symmetric and symmetric under $t_{1} \leftrightarrow t_{2}$ and $t_{3} \leftrightarrow t_{4}$. The first term i.e., $F^{A}\left(\tau_{1}, \tau_{2}, \tau_{3}, \tau_{4}\right)$ is identical to the SYK with Majorana but the second term is new and occurs in the complex fermion model. From [39] we have,

$$
\begin{aligned}
\frac{F^{A}\left(\tau_{1}, \tau_{2}, \tau_{3}, \tau_{4}\right)}{G\left(\tau_{12}\right) G\left(\tau_{34}\right)}= & \alpha_{0} \int_{0}^{\infty} \frac{s d s}{\pi^{2}} \frac{k^{A}\left(\frac{1}{2}+i s\right)}{\operatorname{coth}(\pi s)\left(1-k^{A}\left(\frac{1}{2}+i s\right)\right)} \Psi_{\frac{1}{2}+i s}^{A}(\chi) \\
& +\alpha_{0} \sum_{2 j>0} \frac{2 j-\frac{1}{2}}{\pi^{2}} \frac{k^{A}(2 j)}{1-k^{A}(2 j)} \Psi_{2 j}^{A}(\chi), \\
\frac{F^{S}\left(\tau_{1}, \tau_{2}, \tau_{3}, \tau_{4}\right)}{G\left(\tau_{12}\right) G\left(\tau_{34}\right)}= & \alpha_{0} \int_{0}^{\infty} \frac{s d s}{\pi^{2}} \frac{k^{S}\left(\frac{1}{2}+i s\right)}{\operatorname{coth}(\pi s)\left(1-k^{S}\left(\frac{1}{2}+i s\right)\right)} \Psi_{\frac{1}{2}+i s}^{S}(\chi) \\
& +\alpha_{0} \sum_{2 j+1>0} \frac{2 j+\frac{1}{2}}{\pi^{2}} \frac{k^{S}(2 j+1)}{1-k^{S}(2 j+1)} \Psi_{2 j+1}^{S}(\chi),
\end{aligned}
$$

where

$$
\chi=\frac{\tau_{12} \tau_{34}}{\tau_{13} \tau_{24}},
$$

is the conformal cross ratio and $\Psi^{A}$ and $\Psi^{S}$ are linear combinations of the eigen-functions of the quadratic Casimir. They are antisymmetric, respectively symmetric under the transformation,

$$
\chi \rightarrow \frac{\chi}{\chi-1}
$$

which effectively exchanges the first two or last two arguments of four point function. Finally $k^{A}$ and $k^{S}$ are eigenvalues of the retarded kernels (for antisymmetric and symmetric) which commute with the Casimir.

\subsection{Large $q$ limit}

We now augment this $q$-point interaction with a quadratic coupling term by introducing a chemical potential $\mu$ which couples to the conserved charge $\sum_{i} \psi_{i}^{\dagger} \psi_{i}$. The fermion propagator in the Fourier space derived from the quadratic part of the action is

$$
G_{0}(\mu, \omega)=\frac{1}{i \omega+\mu}
$$


Once we take the interaction terms in the Hamiltonian into account it gives the dressed propagator. In the large $N$ limit, the melonic diagrams contribution dominates. If we also take large $q<N$ limit then the loop corrected propagator is amenable to analytic computations.

$$
G(\mu, \tau)=G_{0}(\mu, \tau)\left(1+\frac{g(\mu, \tau)}{q}+\cdots\right)
$$

where, $G_{0}(\mu, \tau)$ is the free propagator in real space. To compute the two-point function in the interacting theory one sets up the Schwinger-Dyson equation and seeks a solution in the

large $q$ limit. This Schwinger-Dyson equation at finite inverse temperature $\beta$ can be cast in the form of a differential equation

$$
\left(\partial_{\tau}-\mu\right)^{2}\left[G_{0}(\mu, \tau) g(\mu, \tau)\right]=\frac{q J^{2} G_{0}(\mu, \tau)}{(2+2 \cosh (\mu \beta))^{q / 2-1}} \exp \left(\frac{1}{2}\{g(\mu, \tau)+g(\mu,-\tau)\}\right) .
$$

The solution to this equation in given by[40]

$$
e^{g(\mu, \tau)}=\frac{\cos ^{2}\left(\frac{\pi \nu}{2}\right)}{\cos ^{2}\left(\pi \nu\left(\frac{|\tau|}{\beta}-\frac{1}{2}\right)\right)}, \quad \text { where } \quad \beta \mathcal{J}_{\text {eff }}=\frac{\pi \nu}{\cos \left(\frac{\pi \nu}{2}\right)}
$$

where $\mathcal{J}_{\text {eff }}$ is defined in (1.4).

\section{Correlation Functions}

Let us begin with the short time i.e., $\tau_{1}-\tau_{2}=\tau_{12} \rightarrow 0$ limit of the four point function both for the symmetric and anti-symmetric case,

$$
\begin{aligned}
& F^{A}\left(\tau_{1}, \tau_{2}, \tau_{3}, \tau_{4}\right)=G\left(\tau_{12}\right) G\left(\tau_{34}\right) \sum_{n=1}^{\infty} c_{n}^{2}\left(\frac{\left|\tau_{12} \tau_{34}\right|}{\left|\tau_{13} \tau_{14}\right|}\right)^{h_{n}} \\
& F^{S}\left(\tau_{1}, \tau_{2}, \tau_{3}, \tau_{4}\right)=G\left(\tau_{12}\right) G\left(\tau_{34}\right) \sum_{n=1}^{\infty} \tilde{c}_{n}^{2}\left(\frac{\left|\tau_{12} \tau_{34}\right|}{\left|\tau_{13} \tau_{14}\right|}\right)^{h_{n}}
\end{aligned}
$$

When we calculate the the six point function of the complex fermions we go to different short time limits, where the correlation function take some effective form. In the triple short time limit we calculate it as an effective three point function of the fermion bi-linear operators. This way one can compute the correlation function near points where different arguments approach each other yielding poles and by the property of being analytic everywhere else we get the full contribution.

In the remaining part of this article we calculate the $O\left(1 / N^{2}\right)$ coefficient of the six point function with respect to the $1 / N$ expansion. To this order there are contributions from the contact diagrams as well planar diagrams. We will now write down the corresponding expressions:

$$
\mathcal{S}=\mathcal{S}_{1}+\mathcal{S}_{2}+\tilde{\mathcal{S}}_{1}+\tilde{\mathcal{S}}_{2}
$$


Here the contributions of $\mathcal{S}_{1}$ (contact) and $\mathcal{S}_{2}$ (planar) are exactly same as in[41], namely the result for the Majorana fermions. In case of the SYK model with complex fermions, if we demand time reversal invariance, (since the Hamiltonian is itself time reversal invariant) we have only two other contributions. Now,

$$
\frac{\tilde{\mathcal{S}}_{1}}{90}=(q-1)(q-2) J^{2} \iint_{-\infty}^{\infty} d \tau_{a} d \tau_{b} G\left(\tau_{a b}\right)^{q-3} F^{S}\left(\tau_{1}, \tau_{2}, \tau_{a}, \tau_{b}\right) F^{A}\left(\tau_{3}, \tau_{4}, \tau_{a}, \tau_{b}\right) F^{S}\left(\tau_{5}, \tau_{6}, \tau_{a}, \tau_{b}\right),
$$

is the contact diagram contribution. Here, we have written only one particular assignment of the arguments; there are other possible assignments whose contributions account for the factor of 1/90 on the left hand side. There are total 90 possible independent configurations. We will use same symbol $h$ to denote the conformal weight of the bi-linear operators both for $F^{A}$ and $F^{S}$, although the values are different for the two: for $F^{A} \Rightarrow h_{n}=2 n+1+2 \Delta+O\left(\frac{1}{k}\right)$, and for $F^{S} \Rightarrow h_{n}=2 n+2 \Delta+O\left(\frac{1}{k}\right)$. Also we have,

$$
\frac{\tilde{\mathcal{S}}_{2}}{90}=\int_{-\infty}^{\infty} d \tau_{a} d \tau_{b} d \tau_{c} \mathcal{F}_{a m p}^{S}\left(\tau_{1}, \tau_{2}, \tau_{a}, \tau_{b}\right) \mathcal{F}_{a m p}^{A}\left(\tau_{3}, \tau_{4}, \tau_{b}, \tau_{c}\right) \mathcal{F}_{a m p}^{S}\left(\tau_{5}, \tau_{6}, \tau_{c}, \tau_{a}\right)
$$

where,

$$
\mathcal{F}_{\text {amp }}^{S}\left(\tau_{1}, \tau_{2}, \tau_{3}, \tau_{4}\right)=J^{2} \int d \tau_{0} F^{S}\left(\tau_{1}, \tau_{2}, \tau_{3}, \tau_{0}\right) G\left(\tau_{40}\right)^{q-1}
$$

Using the Selberg integrals in its special and general forms, one obtains:

$$
\mathcal{F}_{a m p}^{S}\left(\tau_{1}, \tau_{2}, \tau_{3}, \tau_{4}\right)=G\left(\tau_{12}\right) \sum_{n} \tilde{c}_{n}^{2} \tilde{\xi}_{n} \operatorname{sgn}\left(\tau_{12}\right) \operatorname{sgn}\left(\tau_{43}\right) \frac{\left|\tau_{12}\right|^{h_{n}}\left|\tau_{34}\right|^{h_{n}-1}}{\left|\tau_{24}\right|^{h_{n}+1-2 \Delta}\left|\tau_{23}\right|^{h_{n}-1+2 \Delta}} \cdot
$$

Using the short time expansion of four point amplitudes, we get:

$$
\begin{gathered}
\frac{\tilde{S}_{1}}{90}=b^{q}(q-1)(q-2) J^{2} \sum_{n, m, k} \tilde{c}_{n} c_{m} \tilde{c}_{k}\left|\tau_{12}\right|^{h_{n}}\left|\tau_{34}\right|^{h_{m}}\left|\tau_{56}\right|^{h_{k}} G\left(\tau_{12}\right) G\left(\tau_{34}\right) G\left(\tau_{56}\right) I_{n m k}^{(1)}, \\
\frac{\tilde{S}_{2}}{90}=b^{q}(q-1)(q-2) J^{2} \sum_{n, m, k} \tilde{c}_{n} c_{m} \tilde{c}_{k} \tilde{\xi}_{n} \xi_{m} \tilde{\xi}_{k}\left|\tau_{12}\right|^{h_{n}}\left|\tau_{34}\right|^{h_{m}}\left|\tau_{56}\right|^{h_{k}} \\
\times G\left(\tau_{12}\right) G\left(\tau_{34}\right) G\left(\tau_{56}\right) I_{n m k}^{(2)},
\end{gathered}
$$

where explicit expressions of the constants $c, \xi, \tilde{c}, \tilde{\xi}$ are given in appendix A. The integrals $I^{(1)}$ and $I^{(2)}$ are given by

$$
\begin{aligned}
I_{n m k}^{(1)}\left(\tau_{1}, \tau_{3}, \tau_{5}\right) & =\operatorname{sgn}\left(\tau_{12}\right) \operatorname{sgn}\left(\tau_{56}\right) \int_{-\infty}^{\infty} d \tau_{a} d \tau_{b} \frac{\operatorname{sgn}\left(\tau_{1 a} \tau_{1 b} \tau_{5 a} \tau_{5 b}\right)\left|\tau_{a b}\right|^{h_{n}+h_{m}+h_{k}-2}}{\left|\tau_{1 a}\right|^{h_{n}}\left|\tau_{1 b}\right|^{h_{n}}\left|\tau_{3 a}\right|^{h_{m}}\left|\tau_{3 b}\right|^{h_{m}}\left|\tau_{5 a}\right|^{h_{k}}\left|\tau_{5 b}\right|^{h_{k}}}, \\
I_{n m k}^{(2)}\left(\tau_{1}, \tau_{3}, \tau_{5}\right)= & -\operatorname{sgn}\left(\tau_{12}\right) \operatorname{sgn}\left(\tau_{56}\right) \int_{-\infty}^{\infty} d \tau_{a} d \tau_{b} d \tau_{c}\left[\frac{\operatorname{sgn}\left(\tau_{3 b}\right) \operatorname{sgn}\left(\tau_{3 c}\right)}{\left|\tau_{3 c}\right|^{h_{m}-1+2 \Delta}\left|\tau_{3 a}\right|^{h_{m}+1-2 \Delta}}\right. \\
& \left.\times \frac{\operatorname{sgn}\left(\tau_{a b}\right) \operatorname{sgn}\left(\tau_{b c}\right)\left|\tau_{a b}\right|^{h_{n}-1}\left|\tau_{c a}\right|^{h_{m}-1}\left|\tau_{b c}\right|^{h_{k}-1}}{\left|\tau_{1 a}\right|^{h_{n}-1+2 \Delta}\left|\tau_{1 b}\right|^{h_{n}+1-2 \Delta}\left|\tau_{5 b}\right|^{h_{k}-1+2 \Delta}\left|\tau_{5 c}\right|^{h_{k}+1-2 \Delta}}\right] .
\end{aligned}
$$


The integral (3.8), can be simplified by the change of variables, $\tau_{a} \rightarrow \tau_{1}-\left(1 / \tau_{a}\right)$, and $\tau_{b} \rightarrow$ $\tau_{1}-\left(1 / \tau_{b}\right)$. The simplification is done by first decomposing the integral into sums of integrals. Namely the integration from $-\infty$ to $\infty$ will be written as a sum of two, an integral from $-\infty$ to 0 and an integral from 0 to $\infty$. We implement the change of variables on each fragment separately, simplify each of them before recombining them back. At the end of this exercise, we get

$$
\begin{aligned}
I_{n m k}^{(1)}\left(\tau_{1}, \tau_{3}, \tau_{5}\right)=\operatorname{sgn}\left(\tau_{12}\right) \operatorname{sgn}\left(\tau_{56}\right) & \int_{-\infty}^{\infty} d \tau_{a} d \tau_{b}\left[\frac{1}{\left|\tau_{31}\right|^{2 h_{m}}\left|\tau_{51}\right|^{2 h_{k}}}\right. \\
& \left.\times \frac{\left|\tau_{a b}\right|^{h_{n}+h_{m}+h_{k}-2} \operatorname{sgn}\left(\tau_{51} \tau_{a}+1\right) \operatorname{sgn}\left(\tau_{51} \tau_{b}+1\right)}{\left.\left|\tau_{a}+\frac{1}{\tau_{31}}\right|{ }^{h_{m}}\left|\tau_{b}+\frac{1}{\tau_{31}}\right|\right|_{m}\left|\tau_{a}+\frac{1}{\tau_{51}}\right| h_{k}\left|\tau_{b}+\frac{1}{\tau_{51}}\right|^{h_{k}}}\right] .
\end{aligned}
$$

These change of variables are followed up by another pair of change of variables which are carried out in a sequential manner. We will first implement $\tau_{a} \rightarrow \tau_{a}-\left(1 / \tau_{31}\right)$, and $\tau_{b} \rightarrow$ $\tau_{b}-\left(1 / \tau_{31}\right)$ and then we will rescale the integration variables $\tau_{a} \rightarrow\left(\tau_{53} \tau_{a}\right) /\left(\tau_{31} \tau_{51}\right)$ and $\tau_{b} \rightarrow\left(\tau_{53} \tau_{b}\right) /\left(\tau_{31} \tau_{51}\right)$.

$$
\begin{aligned}
& I_{n m k}^{(1)}\left(\tau_{1}, \tau_{3}, \tau_{5}\right)=\frac{\operatorname{sgn}\left(\tau_{12}\right) \operatorname{sgn}\left(\tau_{56}\right)}{\left|\tau_{31}\right|^{h_{n}+h_{m}-h_{k}}\left|\tau_{51}\right|^{h_{n}+h_{k}-h_{m}}\left|\tau_{53}\right|^{h_{k}+h_{m}-h_{n}}} \times \tilde{I}_{n m k}^{(1)}\left(h_{n}, h_{m}, h_{k}\right), \\
& \tilde{I}_{n m k}^{(1)}\left(h_{n}, h_{m}, h_{k}\right)=\int_{-\infty}^{\infty} d \tau_{a} d \tau_{b} \frac{\left|\tau_{a b}\right|^{h_{n}+h_{m}+h_{k}-2} \operatorname{sgn}\left(\tau_{a}-1\right) \operatorname{sgn}\left(\tau_{b}-1\right)}{\left|\tau_{a}\right|^{h_{m}}\left|\tau_{b}\right|^{h_{m}}\left|1-\tau_{a}\right|^{h_{k}}\left|1-\tau_{b}\right|^{h_{k}}}=\tilde{S}_{2,2}^{\text {full }}(\alpha, \beta, \gamma),
\end{aligned}
$$

where, $\alpha=-h_{n}+1, \beta=-h_{k}+1$, and $\gamma=\frac{h_{n}+h_{m}+h_{k}}{2}-1$.

As in [41], we divide the Selberg integral, $\tilde{S}_{2,2}^{\text {full }}$, into different parts. This is achieved by decomposing the integral into three pieces $[-\infty, 0],[0,1]$ and $[1, \infty]$ for each integration variable. This results in six Selberg integrals with appropriately modified arguments. Carefully keeping track of the signs, gives

$$
\begin{aligned}
\tilde{S}_{2,2}^{\text {full }}(\alpha, \beta, \gamma) & =S_{2,2}(\alpha, \beta, \gamma)+S_{2,2}(1-\alpha-\beta-2 \gamma, \beta, \gamma) \\
& +S_{2,2}(1-\alpha-\beta-2 \gamma, \alpha, \gamma)+2 S_{2,1}(1-\alpha-\beta-2 \gamma, \alpha, \gamma) \\
& -2 S_{2,1}(\alpha, \beta, \gamma)-2 S_{2,1}(\alpha, 1-\alpha-\beta-2 \gamma, \gamma) .
\end{aligned}
$$

The generalized Selberg integrals and some important results which are used above are given in [41], but for completeness we give the relevant definitions here

$$
\begin{aligned}
& S_{n, n}(\alpha, \beta, \gamma)=\int_{[0,1]^{n}} d \tau_{1} \ldots d \tau_{n} \prod_{i=1}^{n}\left|\tau_{i}\right|^{\alpha-1}\left|1-\tau_{i}\right|^{\beta} \prod_{i<j}\left|\tau_{i j}\right|^{2 \gamma} \\
& S_{n, p}(\alpha, \beta, \gamma)=\int_{[0,1]^{p}} \int_{[1, \infty)^{n-p}} d \tau_{1} \ldots d \tau_{n} \prod_{i=1}^{n}\left|\tau_{i}\right|^{\alpha-1}\left|1-\tau_{i}\right|^{\beta} \prod_{i<j}\left|\tau_{i j}\right|^{2 \gamma} .
\end{aligned}
$$

In a similar fashion one can manipulate $I_{n m k}^{(2)}$ to bring it in a form of the conformal three point function. This computation, however, is considerably more involved so we instead do 
the analysis in the large $q$. The $I_{n m k}^{(2)}$ in our case differs from that obtained in [41] by only the sgn functions while the rest of the integrand has exactly the same form. So for us also at large $q, I_{n m k}^{(2)}$ takes the form,

$$
I_{n m k}^{(2)}\left(\tau_{1}, \tau_{2}, \tau_{3}\right) \approx \frac{\tilde{s}_{n m k}^{(2)}}{\left|\tau_{31}\right|^{h_{n}+h_{m}-h_{k}}\left|\tau_{51}\right|^{h_{n}+h_{k}-h_{m}}\left|\tau_{53}\right|^{h_{k}+h_{m}-h_{n}}}+\cdots
$$

In our case of course $\tilde{s}_{n m k}^{(2)}$ is different from $s_{n m k}^{(2)}$ obtained by Gross and Rosenhaus[41].

\section{Away from the Conformal Limit}

In this section we carry out the calculation of correlation functions away from the conformal IR fixed point. In our earlier work [40] we studied the effect of introducing a chemical potential $\mu$, in the SYK-model with complex fermions. We found that a non-zero $\mu$ takes us away from the conformal limit since it explicitly introduces a scale in the problem. The effect of introduction of this scale parameter is reflected in the chaotic behavior of the model, namely, it brings down the value of the Lyapunov exponent. We computed the required quantities and studied the maximally chaotic mode(in the large $q$ limit where things can be handled analytically).

We write below the relevant expressions in the large $q$ limit. The two point function(to the leading order in large $N$ ) in the region $\tau>0$ is given by,

$$
G(\mu, \tau)=G_{0}(\mu, \tau)\left(1+\frac{1}{q} \log \left(\frac{\cos \left(\frac{\pi \nu}{2}\right)}{\cos \left[\pi \nu\left(\frac{1}{2}-\frac{\tau}{\beta}\right)\right]}\right)+. .\right),
$$

where,

$$
\begin{aligned}
& G_{0}(\mu, \tau)=-\frac{e^{\mu \tau}}{e^{\mu \beta}+1}, 0 \leq \tau \leq \beta, \\
& G_{0}(\mu, \tau)=\frac{e^{\mu \tau}}{e^{-\mu \beta}+1},-\beta \leq \tau \leq 0 .
\end{aligned}
$$

The above relation can be written in a compact manner by,

$$
G_{0}(\mu, \tau)=-\operatorname{sgn}(\tau) \frac{e^{\mu \tau}}{e^{\mu \beta \operatorname{sgn}(\tau)}+1}, \quad 0 \leq \tau \leq \beta
$$

We now aim at calculating the enhanced contribution to the four point function slightly away from the conformal limit with the chemical potential $\mu$. Note that since we want to be slightly away from the IR, we will keep $\mu \beta$ to be small and expand all functions in this variable. Then it can be interpreted that we move slightly away from the IR by turning on a small chemical potential.

To this end we need to first calculate the shift in the eigenvalue of the Kernel for the $h=2$ mode. For this we incorporate the technique used in [4]. We begin with the equation,

$$
K \Psi=k \Psi, \quad \Rightarrow \quad \iint K\left(\tau_{1}, \tau_{2}, \tau_{3}, \tau_{4}\right) \Psi\left(\tau_{3}, \tau_{4}\right) d \tau_{3} d \tau_{4}=k \Psi\left(\tau_{1}, \tau_{2}\right) .
$$


The Kernel is given by,

$$
K\left(\tau_{1}, \tau_{2}, \tau_{3}, \tau_{4}\right)=-(-1)^{q / 2} J^{2}(q-1) G\left(\mu, \tau_{13}\right) G\left(\mu,-\tau_{24}\right) G\left(\mu, \tau_{34}\right)^{q / 2-1} G\left(\mu,-\tau_{34}\right)^{q / 2-1}
$$

We will work in the large $q$ limit. Substituting the Kernel in equation (4.5) gives

$$
\begin{aligned}
-q J^{2} \int d \tau_{3} d \tau_{4} & \frac{\operatorname{sgn}\left(\tau_{13}\right) \operatorname{sgn}\left(\tau_{24}\right) e^{\mu \tau_{13}} e^{-\mu \tau_{24}}}{\left(e^{\mu \beta \operatorname{sgn}\left(\tau_{13}\right)}+1\right)\left(e^{-\mu \beta \operatorname{sgn}\left(\tau_{24}\right)}+1\right)} \frac{\cos ^{2}\left(\frac{\pi \nu}{2}\right)}{\sin ^{2}\left(\frac{\tilde{x}_{34}}{2}\right)} \\
& \times \frac{\Psi\left(\tau_{3}, \tau_{4}\right)}{\left\{\left(e^{\mu \beta \operatorname{sgn}\left(\tau_{34}\right)}+1\right)\left(e^{-\mu \beta \operatorname{sgn}\left(\tau_{34}\right)}+1\right)\right\}^{q / 2-1}}=k \Psi\left(\tau_{1}, \tau_{2}\right),
\end{aligned}
$$

where $\nu$ is defined in (2.11) and $\tilde{x}_{i j}=\frac{2 \pi \nu \tau_{i j}}{\beta}+\pi(1-\nu)$. Multiplying (4.7) by $e^{-\mu \tau_{12}}$ on both sides of the equation, and differentiating twice, once with respect to $\tau_{1}$ and once with respect to $\tau_{2}$ gives,

$$
\partial_{\tau_{1}} \partial_{\tau_{2}}\left(\frac{\operatorname{sgn}\left(\tau_{13}\right) \operatorname{sgn}\left(\tau_{24}\right)}{\left(e^{\mu \beta \operatorname{sgn}\left(\tau_{13}\right)}+1\right)\left(e^{-\mu \beta \operatorname{sgn}\left(\tau_{24}\right)}+1\right)}\right)=4 \delta\left(\tau_{13}\right) \delta\left(\tau_{24}\right)
$$

Using the parametrization $k=\frac{2}{h(h-1)}$ eq.(4.7) reduces to,

$$
-\frac{q J^{2} \cos ^{2}\left(\frac{\pi \nu}{2}\right)}{(2+2 \cosh (\mu \beta))^{q / 2-1}} \times \frac{e^{-\mu \tau_{12}}}{\sin ^{2}\left(\frac{\tilde{x}_{12}}{2}\right)} \Psi\left(\tau_{1}, \tau_{2}\right)=\frac{2}{h(h-1)} \partial_{\tau_{1}} \partial_{\tau_{2}}\left(e^{-\mu \tau_{12}} \Psi\left(\tau_{1}, \tau_{2}\right)\right)
$$

If we substitute $\Psi\left(\tau_{1}, \tau_{2}\right)=e^{\mu \tau_{12}} e^{-i n\left(\tau_{1}+\tau_{2}\right)} \psi_{n}\left(\tau_{12}\right)$ then after some manipulation of eq.(4.9) (also using (2.11)) we arrive at the differential equation,

$$
\left[n^{2}+4 \partial_{x}^{2}-\frac{\nu^{2} h(h-1)}{\sin ^{2}\left(\frac{\tilde{x}}{2}\right)}\right] \psi_{n}(x)=0 .
$$

Here, $x=\frac{2 \pi \tau}{\beta}$ and we have suppressed the subscript on $\tau$ since everything is now a function of the time difference $\tau_{12}$.

The solution to this equation with appropriate boundary condition is well known. In fact this is the same equation as obtained in [4]. The solution is given by, (with $\tilde{n}=n / \nu$ )

$$
\begin{aligned}
& \psi_{h, n}(x)=\left(\sin \frac{\tilde{x}}{2}\right)_{2}^{h} F_{1}\left(\frac{h-\tilde{n}}{2}, \frac{h+\tilde{n}}{2}, \frac{1}{2} ; \cos ^{2}\left(\frac{\tilde{x}}{2}\right)\right), \quad n=\text { even } \\
& \psi_{h, n}(x)=\cos \frac{\tilde{x}}{2}\left(\sin \frac{\tilde{x}}{2}\right)_{2}^{h} F_{1}\left(\frac{h-\tilde{n}+1}{2}, \frac{h+\tilde{n}+1}{2}, \frac{3}{2} ; \cos ^{2}\left(\frac{\tilde{x}}{2}\right)\right), n=\text { odd } .
\end{aligned}
$$

The quantization condition on $h$ is obtained by demanding that the wave function vanishes at $x=0$, i.e., $\tilde{x}=\pi(1-\nu)$. As we approach the conformal limit $\nu \rightarrow 1$ this solution actually diverges for generic values of $h$ near 2 (we are interested in the $h=2$ eigenfunctions). But we want values of $h$ such that the solutions are finite or vanishing, so the first or second argument of the hypergeometric has to be a negative integer. This gives the quantization of $h$ near 2 to be,

$$
h_{n}=2+|\tilde{n}|-|n|, \quad h_{n}=2+|n|\left(\frac{1-\nu}{\nu}\right)
$$


This gives the shift in the eigenvalue $k=\frac{2}{h(h-1)}$ to be,

$$
k(2, n)=1-\frac{3|n|}{2}(1-\nu)+\left(\frac{7 n^{2}}{4}-\frac{3|n|}{2}\right)(1-\nu)^{2}+\ldots
$$

This result is identical to the shift obtained in [4], only difference being that $\nu$ now depends on the effective coupling $\beta \mathcal{J}_{\text {eff }}$ instead of $\beta \mathcal{J}$.

\subsection{The enhanced four point contribution}

Let us now look at the four point function and use the above result to figure out the enhanced contribution for the Schwarzian mode slightly away from the conformal limit. We begin with the expansion of the four point function in the basis of eigenfunctions of the Kernel, (using the variable $\theta=\frac{2 \pi \tau}{\beta}$ on the thermal circle and the period becomes $2 \pi$ )

$$
\frac{\mathcal{F}\left(\theta_{1}, \theta_{2}, \theta_{3}, \theta_{4}\right)}{G\left(\theta_{12}\right) G\left(\theta_{34}\right)}=2 \sum_{h, n} \frac{k(h, n)}{1-k(h, n)} \Psi_{h, n}^{\text {exact }}\left(\theta_{1}, \theta_{2}\right) \Psi_{h, n}^{\text {exact } *}\left(\theta_{3}, \theta_{4}\right)
$$

To find the enhanced contribution of the Schwarzian or $h=2$ mode we use the eigenfunction of the Casimir for $h=2$ and the shifted eigenvalue in the denominator. In the numerator we just use the eigenvalue with $h=2$ in the IR. This is done to ensure that we are only slightly away from the conformal limit driven by introducing a small chemical potential. Here we will use all results for the large $q$ limit,

$$
\frac{\mathcal{F}\left(\theta_{1}, \theta_{2}, \theta_{3}, \theta_{4}\right)}{G\left(\theta_{12}\right) G\left(\theta_{34}\right)}=\frac{2 \beta \mathcal{J}_{\text {eff }}}{\pi^{2}} \sum_{|n| \geq 2} \frac{e^{i n\left(y^{\prime}-y\right)}}{n^{2}\left(n^{2}-1\right)}\left[\frac{\sin \left(\frac{n x}{2}\right)}{\tan \left(\frac{x}{2}\right)}-n \cos \left(\frac{n x}{2}\right)\right]\left[\frac{\sin \left(\frac{n x^{\prime}}{2}\right)}{\tan \left(\frac{x^{\prime}}{2}\right)}-n \cos \left(\frac{n x^{\prime}}{2}\right)\right] .
$$

Here,

$$
x=\theta_{1}-\theta_{2}, \quad x^{\prime}=\theta_{3}-\theta_{4}, \quad y=\frac{\theta_{1}+\theta_{2}}{2}, \quad y^{\prime}=\frac{\theta_{3}+\theta_{4}}{2} .
$$

We have used $1-\nu \sim \frac{2}{\beta \mathcal{J}_{\text {eff }}}$ for large $\beta \mathcal{J}_{\text {eff. }}$

We will now carry out the sum over $n$. The final expression after all simplifications is complicated, and to get some sensible result for the six point function using these results we resort to doing numerical computation. That is, we carry out the integration numerically to see the behavior of the six point function. We also deduce the chaotic behavior of the OTO six point correlator even though we do not have an analytic result.

\subsection{The "Contact" and and "Planar" diagrams}

What we want to now claim is that among the Contact diagrams and Planar diagrams, which contribute to the six point function at leading order in $\sim 1 / N$, the contact diagrams dominates the planar ones by an order $q^{4}$, for the enhanced non-conformal mode contribution to the four point function. So at large $q$, the contact ones dominate over the planar ones, and hence we will look at only the former. But let us show a brief argument for why that is true. 
The contact contribution goes as,

$S_{c}=(q-1)(q-2) J^{2} \int d \tau_{a} d \tau_{b} G\left(\tau_{a b}\right)^{\frac{q}{2}-3} G\left(-\tau_{a b}\right)^{\frac{q}{2}} \mathcal{F}\left(\tau_{1}, \tau_{2}, \tau_{a}, \tau_{b}\right) \mathcal{F}\left(\tau_{3}, \tau_{4}, \tau_{a}, \tau_{b}\right) \mathcal{F}\left(\tau_{5}, \tau_{6}, \tau_{a}, \tau_{b}\right)$

While the planar contribution goes as,

$$
\mathcal{S}_{p}=\int_{-\infty}^{\infty} d \tau_{a} d \tau_{b} d \tau_{c} \mathcal{F}_{\mathrm{amp}}\left(\tau_{1}, \tau_{2}, \tau_{a}, \tau_{b}\right) \mathcal{F}_{\mathrm{amp}}\left(\tau_{4}, \tau_{3}, \tau_{c}, \tau_{a}\right) \mathcal{F}_{\mathrm{amp}}\left(\tau_{5}, \tau_{6}, \tau_{b}, \tau_{c}\right)
$$

where,

$$
\mathcal{F}_{\mathrm{amp}}\left(\tau_{1}, \tau_{2}, \tau_{3}, \tau_{4}\right)=-\int_{0}^{\beta} d \tau_{0} \mathcal{F}\left(\tau_{1}, \tau_{2}, \tau_{3}, \tau_{0}\right) \int \frac{d \omega_{4}}{2 \pi} e^{-i \omega_{4} \tau_{40}} \frac{1}{G\left(\mu, \omega_{4}\right)}
$$

is the amputated four point function. We can use the SD equations to write,

$$
\frac{1}{G\left(\mu, \omega_{4}\right)}=-i \omega_{4}+\mu-\Sigma\left(\mu, \omega_{4}\right)
$$

Since we are working at finite temperature, we have to do a Matsubara sum. Notice that the $i \omega+\mu$ term has no poles, so when we evaluate the sum using the contour integration prescription, this part vanishes and we are left with,

$$
\begin{aligned}
& \mathcal{F}_{\text {amp }}\left(\tau_{1}, \tau_{2}, \tau_{3}, \tau_{4}\right)=\int_{0}^{\beta} d \tau_{0} \mathcal{F}\left(\tau_{1}, \tau_{2}, \tau_{3}, \tau_{0}\right) \Sigma\left(\mu, \tau_{40}\right) \\
& \mathcal{F}_{\text {amp }}\left(\tau_{1}, \tau_{2}, \tau_{3}, \tau_{4}\right)=J^{2} \int_{0}^{\beta} d \tau_{0} \mathcal{F}\left(\tau_{1}, \tau_{2}, \tau_{3}, \tau_{0}\right) G\left(\tau_{40}\right)^{q / 2-1} G\left(-\tau_{40}\right)^{q / 2} .
\end{aligned}
$$

Now we can convert the $\tau$ integrals to $\theta$ integrals via appropriate scaling and we get,

$$
S_{c} \sim \frac{q\left(\beta \mathcal{J}_{\text {eff }}\right)^{3}}{(2 \pi)^{2}}
$$

where,

$$
\mathcal{F}_{\text {amp }}\left(\tau_{1}, \ldots, \tau_{4}\right) \sim \frac{\beta \mathcal{J}_{\text {eff }}}{2 \pi q \beta}
$$

In terms of the $\theta$ variable we have,

$$
\mathcal{F}\left(\theta_{i}, \theta_{j}, \theta_{a}, \theta_{b}\right) \sim \beta \mathcal{J}_{\text {eff }} G\left(\theta_{i j}\right) G\left(\theta_{a b}\right)
$$

and in the large $q$ limit, for large but finite $\beta \mathcal{J}_{\text {eff }}$,

$$
\left(G\left(\theta_{a b}\right)\right)^{\frac{q}{2}}\left(G\left(-\theta_{a b}\right)\right)^{\frac{q}{2}} \sim \frac{1}{\left(\beta \mathcal{J}_{\text {eff }}\right)^{2} \sin ^{2}\left(\frac{\theta_{a b}}{2}\right)} .
$$

Here we have put $\nu=1$ inside the sine function which is consistent to the leading order with $\nu \rightarrow 1$ as $\beta \mathcal{J}_{\text {eff }} \rightarrow \infty$. As a consequence the $\left(\beta \mathcal{J}_{\text {eff }}\right)^{2}$ coefficient of the "Contact" diagram as well as the amputated four point function cancels out due to the $\left(\beta \mathcal{J}_{\text {eff }}\right)^{2}$ appearing in the denominator of (4.23). Now since the planar diagram is given by the product of three 
amputated four point functions hence, when we take the product and convert the $\tau$ integrals to $\theta$ integrals in (4.17), we finally get,

$$
\mathcal{S}_{p} \sim \frac{\left(\beta \mathcal{J}_{\text {eff }}\right)^{3}}{q^{3}(2 \pi)^{6}}
$$

Taking the ration of $S_{c}$ with $\mathcal{S}_{p}$ we see that,

$$
\frac{S_{c}}{\mathcal{S}_{p}} \sim(2 \pi)^{4} q^{4} .
$$

So in the large $q$ limit as one can easily see that the contact diagram is far more dominant compared to the planar ones and hence it is justified to consider the contribution of the contact diagrams only.

\subsection{The six point function}

Although one can get an analytic answer for the enhanced four point contribution slightly away from the conformal limit, calculation of the full six point function becomes somewhat messy to carry out analytically. We therefore compute the six point function using numerical methods.

Let us first summarize the results, we will then we state all the relevant values used in carrying out these computations.

- We first compute the six point contribution with three $h=2$ mode keeping all the time arguments to be separate and then we take the short time limit $\theta_{1} \rightarrow \theta_{2}$ or $\theta_{3} \rightarrow \theta_{4}$. We see that the six point function decreases in this limit for various small values of $\mu \beta$ keeping $\beta \mathcal{J}$ fixed at some large value.

- We then reverse the order, that is we first take the triple short time limit and then carry out the integrals numerically for all the possible nonconformal contributions, i.e.,

$$
\mathcal{F}_{h=2} \mathcal{F}_{c} \mathcal{F}_{c}, \quad \mathcal{F}_{h=2} \mathcal{F}_{h=2} \mathcal{F}_{c}, \quad \mathcal{F}_{h=2} \mathcal{F}_{h=2} \mathcal{F}_{h=2}
$$

We find that among the three terms listed above, the first contribution almost vanishes up to any order in $\mu \beta$ that we are working with, whereas the other two terms are small but are of the same order and they go as $(\mu \beta)^{2}$ with small coefficients. These will get corrected as we go to higher orders.

- To benchmark the code we compute $\lambda_{11 k}^{(1)}$ (as was done in [41] for all three conformal modes) for the contact diagrams and plot it against $k$, where for large $k, h_{k}=2 k+1+$ $1 / 2 \Delta+O(1 / k)$. We find the similar fall off behavior at large $k$.

Let us now look at some details of the analysis. One of the things that we have to keep in mind is that we are slightly away from the conformal limit because we have turned on a small 
$\mu \beta$. We need to be careful while working with the conformal modes. Due to explicit scale in the theory, the modes may not be conformal anymore. In other words, the normalized four point contributions of these modes may not be a function of only the cross ratio $\chi$ anymore. However, for small $\beta \mu$, the conformal perturbation theory makes sense and within this limit using the conformal basis is justified.

If we recall the eigenvectors of the Kernel then we see that,

$$
\Psi\left(\theta_{1}, \theta_{2}\right)=e^{\frac{\mu \beta}{2 \pi}\left(\theta_{12}\right)} e^{i n \frac{\theta_{1}+\theta_{2}}{2}} \psi_{n}\left(\theta_{12}\right)
$$

and to obtain the conformal modes one has to go to the IR, do the sum over $n$ in the four point function to obtain the sum over integer values of $h$ as well as the integral over the principle continuous series. One then deforms the contour to pick up the poles at $k_{c}=1$, eigenvalue of the kernel in the conformal limit. In the IR limit the exponential $\mu \beta$ factor becomes equal to 1 , but since it has no $n$ dependence it plays no role when we carry out the sum over $n$. Therefore, slightly away from the conformal limit we will have (small $\mu \beta$ ),

$$
\begin{gathered}
\frac{\mathcal{F}_{h \neq 2}\left(\theta_{1}, \theta_{2}, \theta_{3}, \theta_{4}\right)}{G\left(\theta_{12}\right) G\left(\theta_{34}\right)}=e^{\frac{\mu \beta}{2 \pi}\left(\theta_{12}+\theta_{34}\right)} \sum_{m=1}^{\infty} c_{m}^{2} \chi_{2}^{h_{m}} F_{1}\left(h_{m}, h_{m}, 2 h_{m}, \chi\right) \\
=\sum_{m=1}^{\infty} c_{m}^{2} \chi_{2}^{h_{m}}{ }_{2} F_{1}\left(h_{m}, h_{m}, 2 h_{m}, \chi\right)+\frac{\mu \beta\left(\theta_{12}+\theta_{34}\right)}{2 \pi} \sum_{m=1}^{\infty} c_{m}^{2} \chi_{{ }_{2}}^{h_{m}} F_{1}\left(h_{m}, h_{m}, 2 h_{m}, \chi\right) \\
+\left(\frac{\mu \beta}{2 \pi}\right)^{2} \frac{\left(\theta_{12}+\theta_{34}\right)^{2}}{2 !} \sum_{m=1}^{\infty} c_{m}^{2} \chi^{h_{m}}{ }_{2} F_{1}\left(h_{m}, h_{m}, 2 h_{m}, \chi\right)+\cdots
\end{gathered}
$$

The above expression breaks conformal invariance and this is the four point function we will be working with away from the conformal limit.

Since $\beta \mathcal{J}_{\text {eff }}$ appears as an overall factor we strip of this factor and look at the integrals only. For small $\mu \beta$ this factor is large but finite. $\mu \beta$ is kept to be $\sim 7.4 \times 10^{-4}$.

\subsection{The Short time and OTO behavior of the Six point function}

Since this is a part of the non-conformal piece, we will first compute it by keeping all times different and then taking one time approaching another for example $\tau_{1} \rightarrow \tau_{2}$, and look at how it behaves. In Figure 1, we see the behavior of the six point as we take the short time limit. It is easy to check that when we compute the six point function in the triple short time limit vanishes.

The contribution to the non-conformal piece coming from the product of three $h=2$ modes is shown in Fig. 1. In computing the contribution for one or two $h \neq 2$ modes, we have to be careful as what happens to the "Planar" diagrams as well. For more details see, Appendix B.

For calculating the OTO correlation function we have to first set a specific configuration of operators, which is then analytically continued to real time. However, we do not have the analytic expression so we use the following procedure. We modify the times that are to be 


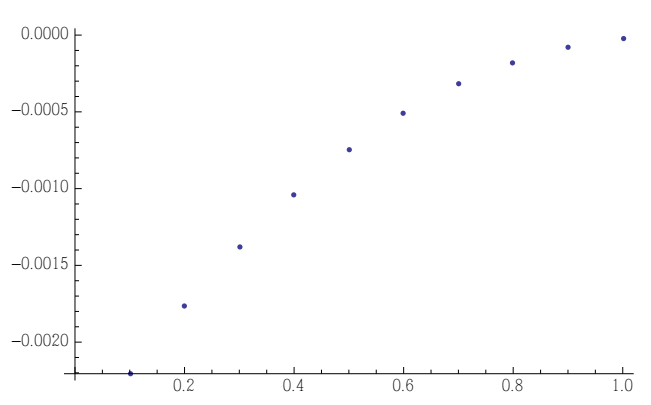

Figure 1. The plot of the six point function vs $\tau_{1}$ as $\tau_{1} \rightarrow \tau_{2}$.

analytically continued keeping their relative positions to be the same. This effectively corresponds to changing only one time independently. We observe the behavior of the correlator and compare it with the behavior of the enhanced four point function (for which we already know that there is a chaotic behavior) under the same operation. From the results plotted in
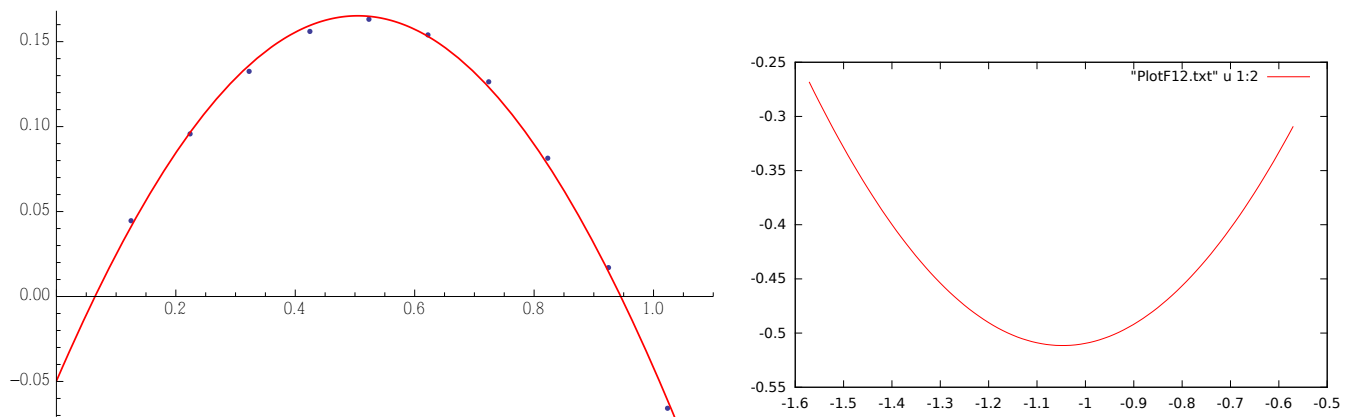

Figure 2. The OTO six point and four point function response for changing $\tau_{1}$.

fig. 1 and fig. 4.4 above it is easy to see that the six point OTO correlation function actually does exhibit chaotic behavior when we analytically continue to real time. The chaos exponent can be estimated by fitting the data with an oscillatory function. We choose an ansatz

$$
a+b \sin (\lambda \tau)+c \cos (\lambda \tau) .
$$

We find that the data is fitted for the following values of $a, b, c$, and $\lambda$,

$$
\begin{aligned}
a & =-0.68904, \\
b & =0.56662, \\
c & =0.63927, \\
\lambda & =1.4364 .
\end{aligned}
$$

The maximal value of the Lyapunov is $3 / 2$ but the fitted value of $\lambda$ is less than the maximal. This is because we are away from the conformal point due to small but non-vanishing value of the chemical potential $\mu$. Here we would like to mention that upto small errors this value of $\lambda$ is in agreement with [42], as the maximal chaos exponent for the six point OTOC. 


\section{Conclusion}

We have computed the fermion six point function in the SYK model with complex fermions in the presence of a non-vanishing chemical potential. We then took triple short time limit of this correlation function so that it appears as a three point function of fermion bilinears. We show that the three point function of fermion bilinears, for $h \neq 2$ modes, have the scaling property of conformal field theory three point function, as is expected as a generalisation of the results of [41] to the complex fermion case. Like in [41], we find that the contribution of the contact three point graphs in the large $q$ limit is subleading compared to that of the planar graphs.

We also compute three point function of fermion bilinears for the $h=2$ mode. This mode is known to break the conformal invariance of the SYK model, both spontaneously as well as explicitly. This mode is known to exhibit chaotic behaviour with the Lyapunov exponent $\lambda_{\mathrm{L}}$ that saturates the chaos bound. The three point function of bilinears in this case has a behaviour different from those of the conformal, i.e., $h \neq 2$ modes. In this case we find that in the large $q$ limit, the contribution of the planar graphs is subleading compared to the contact graphs.

As a future direction to explore further, since the couplings of the SYK model are chosen from random gaussian distributions, it is tempting to ask if one can apply techniques of stochastic quantisation to reconstruct the bulk description. We hope to report on this soon.

Acknowledgments: We would like to thank Subhroneel Chakrabarti for participating in the initial stages of this work and for many discussions. RB would also like to thank Kasi Jaswin for important discussions related to the OTOC behavior. This research was supported in part by the International Centre for Theoretical Sciences (ICTS) during a visit for participating in the program - AdS/CFT at 20 and Beyond (Code: ICTS/adscft20/05) during the course of this work. 


\section{A Appendix A}

In this appendix we collect the expressions of the constants that appear in six point amplitude.

$$
\begin{aligned}
& c_{n}=\frac{2 q}{(q-1)(q-2) \tan (\pi \Delta)} \frac{\left(h_{n}-\frac{1}{2}\right)}{\tan \left(\frac{\pi h_{n}}{2}\right)} \frac{\Gamma^{2}\left(h_{n}\right)}{k_{A}^{\prime}\left(h_{n}\right) \Gamma\left(2 h_{n}\right)}, \\
& \tilde{c}_{n}=\frac{2 q}{(q-1)(q-2) \tan (\pi \Delta)} \frac{\left(h_{n}-\frac{1}{2}\right)}{\cot \left(\frac{\pi h_{n}}{2}\right)} \frac{\Gamma^{2}\left(h_{n}\right)}{k_{S}^{\prime}\left(h_{n}\right) \Gamma\left(2 h_{n}\right)}, \\
& \xi_{n}=b^{q} \pi^{1 / 2} \frac{\Gamma\left(1-\Delta+\frac{h_{n}}{2}\right) \Gamma\left(\frac{1}{2}-\frac{h_{n}}{2}\right) \Gamma(\Delta)}{\Gamma\left(\frac{1}{2}+\Delta-\frac{h_{n}}{2}\right) \Gamma\left(\frac{h_{n}}{2}\right) \Gamma\left(\frac{3}{2}-\Delta\right)}, \\
& \tilde{\xi}_{n}=b^{q} \pi^{1 / 2} \frac{\Gamma\left(\frac{1}{2}-\Delta+\frac{h_{n}}{2}\right) \Gamma\left(1-\frac{h_{n}}{2}\right) \Gamma(\Delta)}{\Gamma\left(\Delta-\frac{h_{n}}{2}\right) \Gamma\left(\frac{1}{2}+\frac{h_{n}}{2}\right) \Gamma\left(\frac{3}{2}-\Delta\right)} .
\end{aligned}
$$

\section{B Appendix B}

If we consider the behavior of $\mathcal{F}_{\text {amp }}$ for the $h=2$ mode as we take two times to be closer to each other, then we find the results shown in Figure 3 below.

As we can see from the graph the value of $\mathcal{F}_{\text {amp }}$ is quite large when the times are well separated. But computing the six point "Planar" diagram in the triple short time limit numerically one finds that the contribution is small i.e of the same order as the "Contact" integrals hence using the fact that the coefficient of the "Contact" diagrams are much dominant over the "Planar" ones, we are justified in considering the contribution of the former only. Although one has to be careful when considering the "Planar" contribution when $h \neq 2$ modes are taken but since the numerics is pretty involved and takes very long time we do not present those results here.

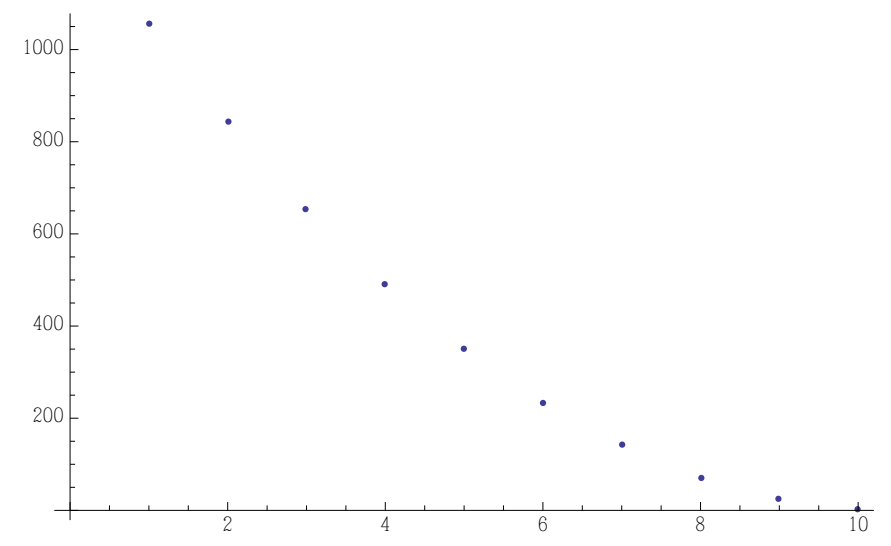

Figure 3. Plot of $\mathcal{F}_{\text {amp }}$ against $\theta_{1}$ as it is taken towards to $\theta_{2}$. Along the $\theta_{1}$ axis we mark the number of steps while the actual interval is $0-1$ in steps of 0.1 


\section{References}

[1] S. H. Strogatz, Nonlinear Dynamics and Chaos. Addison Wesley, Reading, MA, 1994.

[2] S. H. Shenker and D. Stanford, Black holes and the butterfly effect, JHEP 03 (2014) 067, [arXiv: 1306.0622].

[3] A. Y. Kitaev, Entanglement in strongly-correlated quantum matter, . Talk at KITP, University of California, Santa Barbara.

[4] J. Maldacena and D. Stanford, Remarks on the Sachdev-Ye-Kitaev model, Phys. Rev. D94 (2016), no. 10 106002, [arXiv:1604.07818].

[5] J. Engelsöy, T. G. Mertens, and H. Verlinde, An investigation of $A d S_{2}$ backreaction and holography, JHEP 07 (2016) 139, [arXiv:1606.03438].

[6] K. Jensen, Chaos in AdS 2 Holography, Phys. Rev. Lett. 117 (2016), no. 11 111601, [arXiv: 1605.06098].

[7] J. Maldacena, S. H. Shenker, and D. Stanford, A bound on chaos, JHEP 08 (2016) 106, [arXiv: 1503.01409].

[8] J. Maldacena, D. Stanford, and Z. Yang, Conformal symmetry and its breaking in two dimensional Nearly Anti-de-Sitter space, PTEP 2016 (2016), no. 12 12C104, [arXiv: 1606.01857].

[9] P. Nayak, A. Shukla, R. M. Soni, S. P. Trivedi, and V. Vishal, On the Dynamics of Near-Extremal Black Holes, JHEP 09 (2018) 048, [arXiv: 1802.09547].

[10] E. Witten, An SYK-Like Model Without Disorder, arXiv:1610.09758.

[11] G. Mandal, P. Nayak, and S. R. Wadia, Coadjoint orbit action of Virasoro group and two-dimensional quantum gravity dual to SYK/tensor models, JHEP 11 (2017) 046, [arXiv: 1702.04266].

[12] J. Sonner and M. Vielma, Eigenstate thermalization in the Sachdev-Ye-Kitaev model, JHEP 11 (2017) 149, [arXiv:1707.08013].

[13] M. Haque and P. McClarty, Eigenstate Thermalization Scaling in Majorana Clusters: from Integrable to Chaotic SYK Models, arXiv:1711.02360.

[14] D. Stanford and E. Witten, Fermionic Localization of the Schwarzian Theory, JHEP 10 (2017) 008, [arXiv:1703.04612].

[15] M. Berkooz, P. Narayan, M. Rozali, and J. Simon, Higher Dimensional Generalizations of the SYK Model, JHEP 01 (2017) 138, [arXiv:1610.02422].

[16] A. Eberlein, V. Kasper, S. Sachdev, and J. Steinberg, Quantum quench of the Sachdev-Ye-Kitaev Model, Phys. Rev. B96 (2017), no. 20 205123, [arXiv:1706.07803].

[17] J. Murugan, D. Stanford, and E. Witten, More on Supersymmetric and 2d Analogs of the SYK Model, JHEP 08 (2017) 146, [arXiv:1706.05362].

[18] I. R. Klebanov and G. Tarnopolsky, On Large $N$ Limit of Symmetric Traceless Tensor Models, JHEP 10 (2017) 037, [arXiv:1706.00839].

[19] I. Kourkoulou and J. Maldacena, Pure states in the SYK model and nearly-AdS $S_{2}$ gravity, arXiv: 1707.02325. 
[20] J. Erdmenger, M. Flory, M.-N. Newrzella, M. Strydom, and J. M. S. Wu, Quantum Quenches in a Holographic Kondo Model, JHEP 04 (2017) 045, [arXiv:1612.06860].

[21] P. Narayan and J. Yoon, SYK-like Tensor Models on the Lattice, JHEP 08 (2017) 083, [arXiv: 1705.01554].

[22] S. R. Das, A. Jevicki, and K. Suzuki, Three Dimensional View of the SYK/AdS Duality, JHEP 09 (2017) 017, [arXiv: 1704.07208].

[23] G. Turiaci and H. Verlinde, Towards a 2d QFT Analog of the SYK Model, JHEP 10 (2017) 167, [arXiv:1701.00528].

[24] I. R. Klebanov and G. Tarnopolsky, Uncolored random tensors, melon diagrams, and the Sachdev-Ye-Kitaev models, Phys. Rev. D95 (2017), no. 4 046004, [arXiv:1611.08915].

[25] C. Krishnan, K. V. Pavan Kumar, and D. Rosa, Contrasting SYK-like Models, JHEP 01 (2018) 064, [arXiv: 1709.06498].

[26] C. Krishnan, S. Sanyal, and P. N. Bala Subramanian, Quantum Chaos and Holographic Tensor Models, JHEP 03 (2017) 056, [arXiv: 1612.06330].

[27] C. Krishnan and K. V. P. Kumar, Towards a Finite-N Hologram, JHEP 10 (2017) 099, [arXiv: 1706.05364].

[28] N. Callebaut and H. Verlinde, Entanglement Dynamics in 2D CFT with Boundary: Entropic origin of JT gravity and Schwarzian QM, arXiv:1808.05583.

[29] A. Goel, H. T. Lam, G. J. Turiaci, and H. Verlinde, Expanding the Black Hole Interior: Partially Entangled Thermal States in SYK, arXiv:1807.03916.

[30] A. Gaikwad, L. K. Joshi, G. Mandal, and S. R. Wadia, Holographic dual to charged SYK from 3D Gravity and Chern-Simons, arXiv:1802.07746.

[31] S. Choudhury, A. Dey, I. Halder, L. Janagal, S. Minwalla, and R. Poojary, Notes on melonic $O(N)^{q-1}$ tensor models, JHEP 06 (2018) 094, [arXiv: 1707.09352].

[32] S. R. Das, A. Ghosh, A. Jevicki, and K. Suzuki, Space-Time in the SYK Model, JHEP 07 (2018) 184, [arXiv:1712.02725].

[33] K. Bulycheva, I. R. Klebanov, A. Milekhin, and G. Tarnopolsky, Spectra of Operators in Large $N$ Tensor Models, Phys. Rev. D97 (2018), no. 2 026016, [arXiv:1707.09347].

[34] P. Narayan and J. Yoon, Supersymmetric SYK Model with Global Symmetry, JHEP 08 (2018) 159, [arXiv: 1712.02647].

[35] I. R. Klebanov, F. Popov, and G. Tarnopolsky, TASI Lectures on Large N Tensor Models, PoS TASI2017 (2018) 004, [arXiv: 1808.09434].

[36] A. Kitaev and S. J. Suh, The soft mode in the Sachdev-Ye-Kitaev model and its gravity dual, JHEP 05 (2018) 183, [arXiv:1711.08467].

[37] S. R. Das, A. Ghosh, A. Jevicki, and K. Suzuki, Three Dimensional View of Arbitrary q SYK models, JHEP 02 (2018) 162, [arXiv: 1711.09839].

[38] R. A. Davison, W. Fu, A. Georges, Y. Gu, K. Jensen, and S. Sachdev, Thermoelectric transport in disordered metals without quasiparticles: The Sachdev-Ye-Kitaev models and holography, Phys. Rev. B95 (2017), no. 15 155131, [arXiv:1612.00849]. 
[39] K. Bulycheva, A note on the SYK model with complex fermions, JHEP 12 (2017) 069, [arXiv: 1706.07411].

[40] R. Bhattacharya, S. Chakrabarti, D. P. Jatkar, and A. Kundu, SYK Model, Chaos and Conserved Charge, JHEP 11 (2017) 180, [arXiv:1709.07613].

[41] D. J. Gross and V. Rosenhaus, The Bulk Dual of SYK: Cubic Couplings, JHEP 05 (2017) 092, [arXiv: 1702.08016].

[42] P. Basu and K. Jaswin, Higher point otocs and the bound on chaos, arXiv:1809.05331. 